\section{A simple Method for quantifying Triazine Herbicides using Thin-Layer Chromatography and a CCD-Camera}

Prof. Dr. rer. nat. Bernd Spangenberg

Fakultät Maschinenbau und Verfahrenstechnik $(M+V)$

Studiendekan Verfahrenstechnik

Badstraße 24

77652 Offenburg

Tel. 0781 205-101

E-Mail: Spangenberg@fh-offenburg.de
1955: Geboren in Remscheid

Studium der Chemie und Philosophie an der Universität Marburg 1983: Diplom in Chemie, 1987 Promotion über neue Reaktionen von Sulfenen am Pharmazeutischen Institut der Universität Marburg 1988: Eintritt in die E. Scheurich Pharmwerk GmbH als Laborleiter, Leiter der Abteilung analytische Forschung und Entwicklung, stellvertretender Kontrollleiter 1990: Wechsel zur EBULON AG,

Basel als Leiter der Entwicklungsanalytik

1991: Professor an der Hochschule Offenburg, Leiter des Labors für Analytik und Umweltanalytik

Seit 2007: Editor-in-Chief von Journal of Planar Chromatography

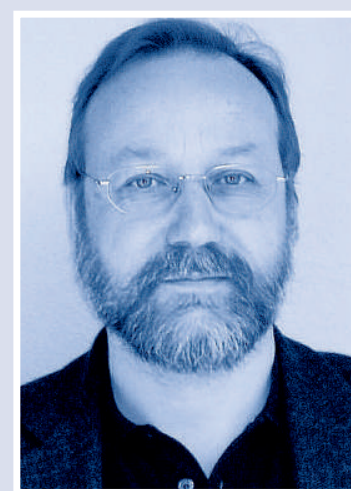

Lehrgebiete: Analytik, Umweltanalytik, Abfall- und Recyclingtechnik

Mitglied des Instituts für Angewandte Forschung (IAF) der Hochschule Offenburg

Forschungsgebiete: Chemometrie, Trenntechniken, Dünnschichtchromatographie

\subsection{A simple Method for quantifying Triazine Herbicides using Thin-Layer Chromatography and a CCD-Camera}

Prof. Dr. rer. nat. Bernd Spangenberg Dipl.-Ing. (FH) Melanie Broszat

Helena Ernst

\section{Introduction}

Modern TLC-scanners can measure in absorption, fluorescence and also in transmittance. TLC-scanners cover the whole wavelength range from 200 up to $1100 \mathrm{~nm}$. The disadvantage of TLC and HPTLC scanners is their high purchase price and maintenance costs. The high price of modern TLC-scanners makes image analysis in thin-layer chromatography (TLC) so interesting [1]. Most TLC-applications are designed to work in the wavelength range from 400 to 800 $\mathrm{nm}$, using human eyes as detectors. Scanning equipment like CCD-cameras (charge coupling device-cameras) or flatbed-scanners working in the visible range are cheaply available and can be used for plate evaluation [1]. The term video-densitometer has also been introduced for such scanning devices.

The advantage of video-densitometric devices is their small size and low weight which make these camera systems portable [2]. The use of a CCD device provides ultraviolet response and a larger dynamic range than a photomultiplier [3, 4]. The evaluation of 2D separations is possible which is not possible with slit-scanners. The principle of CCD-scanning is not restricted to came- ras. A flat-bed scanner can also be used for plate measuring [5]. Commonly used flatbed-scanners illuminate the plate with white light and can scan coloured zones. Even fluorescence can be measured if the flat-bed scanner is equipped with a UV-lamp [6]. The cheapest CCDtechnique for TLC evaluations is to use a hand-scanner [7].

The disadvantage of a video-densitometer is that spectral information is not available. This makes spectral peak identification and spectral peak-purity tests impossible. Otherwise, most substances show no light absorption or fluorescence in the vis-range. To make separation more specific, we recommend a staining step which often makes spectral identification and peak-purity testing unnecessary.

What features should be taken into consideration when buying a CCD-camera? Quantitative video-densitometric measuring needs a detector, which can linearly digitalize light intensity measurements. Double-fold light intensity must result in doubled signal values, which can be checked by changing the measurement time. Therefore double measurement time must result in doubled measured values. The digital resolution of commonly used cameras is 8 bit. A signal is rendered in $2^{8}=256$ different increments (grey levels), which is not sufficient for quantification purposes because at least 12 bit capacity is necessary for quantifying $\left(2^{12}=4096\right.$ increments). CCD-cameras with a resolution of 16 bits are much better because such cameras render $2^{16}=65536$ grey scales. Relatively inexpensive cameras with suitable software thatmeet these requirements are available for astronomy observations. These cameras produce TIFF-pictures, because the TIF-Format (Tagged Image File Format) supports 16 bit data storing.

Although inexpensive flatbed scanners and cheap cameras are not linearly calibrated, it is nevertheless possible to quantify planar chromatography separations. For example the heavy metal complexes cobalt-dithizone and zinc-dithizone can be quantified but the working range covers only a single magnitude due to the non-linear detector [7].

The group of triazine herbicides, which includes atrazine, causes underground water contamination. Atrazine is said to have a carryover, a generally undesirable property for herbicides. In $1952 \mathrm{H}$. Gysin and E. Knüsli synthesized a number of substituted triazines. A first publication appeared in 1955 [8]. Triazine herbicides are colourless compounds detectable only in UV. Triazine herbicides react with chlorine and iodine-starch forming brown-red spots [9] when separated by thin-layer chromatography [8, 10 - 12]. This reaction can be used to quantify TLC-separated triazine herbicides using a CCD-camera.

The purpose of this work is to show that video-densitometric measurements provide a powerful tool for inexpensive quantitative thin layer chromatography. Linear calibration functions over two ma- 
gnitudes and more can be obtained using a linear measuring device in combination with the correct remission theory.

\section{Therory}

In planar chromatography light is used for detecting separated sample spots by illuminating the TLC-plate from the top with light of known intensity. A clean illuminated plate will absorb a share of this illuminating light by the layer. The share of light, which is not absorbed but reflected by the surface, should be $J_{0}$. If this reflected light shows higher intensity than the reflected light (J) from a sample zone, a fraction of light must be absorbed by the sample (the analyte). The difference between these light intensities is absorbed by the analyte and defines the analyte absorption coefficient a.

$$
I_{a b s}=J_{0}-J=a J_{0}
$$

Increasing sample amounts will induce a decreasing light reflection (J). Therefore a transformation algorithm is needed which turns decreasing light intensities into increasing signal values. Ideally there should be a linear relationship between the transformed measurement data and the analyte amount.

With the abbreviation:

$$
R=\frac{J}{J_{0}}
$$

We see that theoretical considerations lead to following equation for transformation purposes that show linearity between the transformed measurement data (TMD) and the absorption coefficient [13].

$$
T M D(k)=k\left(\frac{1}{R}-R\right)+(R-1)
$$

k: backscattering factor $(k \geq 0$ and $k \leq 1)$ a: absorption coefficient

The value of the so called backscattering factor $\mathrm{k}$ is in the range between 0 and 1 and depends on the scattering quality of the stationary phase. In TLC the Kubelka/ Munk theory is often used for evaluation purposes. The Kubelka/Munk theory was first published in the year 1931 and is based on the assumption that half of the scattered flux is directed forwards and half backwards [13]. The backscattering factor in the Kubelka/Munk-theory is $k=1 / 2$ and the correct Kubelka/Munkexpression can be used to obtain linear calibration curves for high analyte concentrations. In trace analysis it is mostly sufficient to use a k-factor $k=1$ for obtaining linearity for calibration curves.

$$
\operatorname{TMD}(k=1)=\frac{1}{R}-1=\frac{a}{1-a}
$$

For $k=0$ no incident light is reflected to the plate top and the resulting expression can be used for fluorescence evaluation [13].

\section{Results and discussion}

For direct video-densitometric evaluation a ST-1603ME CCD camera with 1.56 megapixel from Santa Barbara Instrument Group, Inc., Santa Barbara, USA was used. The camera was mounted with a Kodak KAF-1603ME CCD pixel array containing $1530 \times 1020$ pixel. The array size is $13.8 \times 9.2 \mathrm{~mm}$ with a pixel size of $9 \times 9$ microns. The camera uses a 16 bit A/D converter and a high speed USB interface. The camera was used in combination with a Schneider SKR KMP Xenoplan 28/2,0 - M30,5 lens. For plate evaluation the CCD-array was cooled to $-5^{\circ} \mathrm{C}$. The plate was measured in the dark using two LEDs emitting white light. The time of 6 seconds is necessary to measure the full 16 bit range.

The HPTLC-plate is placed below the camera at a distance of $30 \mathrm{~cm}$. This distance is adjusted so that $8.5 \mathrm{~cm}$ are detected by 1020 pixel providing a resolution of $83.3 \mu \mathrm{m}$ per pixel. A single $\mathrm{mm}$ separation distance is measured by 12 diodes producing 12 data points.

Figure 3.1-1 shows the result of a videodensitometric evaluation of the five triazines atraton, terbumeton, simazine, atrazine and terbutylazine (50 ng each), separated on silica gel with the mobile phase methyl-t-butyl ether and cyclohexane $(1+1, v / v)$. The plate was stained with iodine-starch reagent after treatment with chlorine. For staining the plate was dried in a gentle stream of air for 5 minutes and placed in a chlorine containing chamber for 5 minutes. Chlorine was produced from $10 \mathrm{~mL} \mathrm{KMnO}$ solution $\left(3 \mathrm{~g} \mathrm{KMnO}_{4}\right.$ dissolved in 100 $\mathrm{mL}$ of water) and $10 \mathrm{~mL} \mathrm{HCl}(25 \mathrm{~mL}$ $32 \% \mathrm{HCl}$ dissolved in $50 \mathrm{~mL}$ of water). Five minutes after mixing, the chamber was filled with chlorine and the TLCplate could be placed.

The staining reagent starch-iodine was found to show sufficiently sensitive. To produce the starch-iodine reagent, 800 $\mathrm{mg}$ of potassium iodide was dissolved in $20 \mathrm{~mL}$ of water. $800 \mathrm{mg}$ starch (according to Zulkowsky) was dissolved in 20 $\mathrm{mL}$ of water. Both solutions were mixed and dissolved with $10 \mathrm{~mL}$ of ethanol. The mixture is stable for one day. Redbrown zones are formed on a slightly dark background, if the chlorinated plate is dipped for $1 \mathrm{~s}$ in starch-iodine reagent. The colours are stable for days.

Each stained application band (band width is $7 \mathrm{~mm}$ ) was measured with 64 data points resulting in 64 densitograms. These 64 densitograms measured from each band-wise application were combined in a single densitogram. This data averaging improves the signal-to-noise ratio by a factor of 8 in comparison to the signal-to noise ratio of a densitogram registered by only a single pixel. It's important for all tracks to be evaluated with the same number of measurement data located in the centre of the application band. To achieve reliable evalua-
Fig. 3.1-1: Plotted is the separation of atraton, terbumenton, simazine, atrazine and terbutylazine (50 ng each, from left to right) on silica gel evaluated with expression (4). Als eluent methyl-tbutyl ether, cyclohexane $(1+1, v / v)$ was used for a separation over a distance of $70 \mathrm{~mm}$

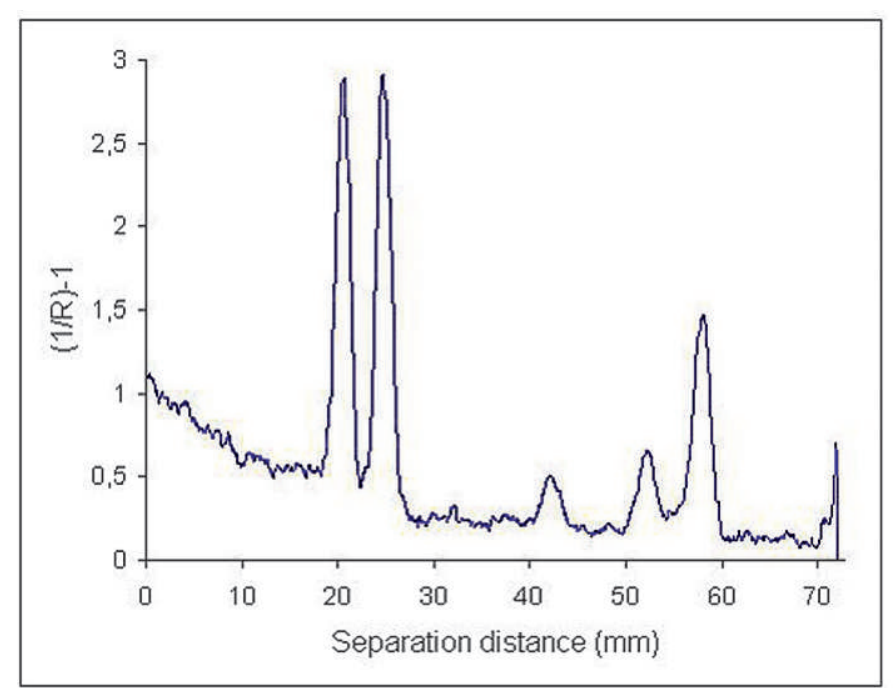


tion, all tracks must also be evaluated using the same position within the track and the same evaluation widths.

Figure 3.1-2 shows the brightest plate area in bits plotted against the measurement time. The plot emphasizes that the camera measures light intensities in a directly linear way. The response curve is not logarithmic as is the case in all commonly used cameras in our daily life.

A sample application was measured at six different time intervals ( 1 to 6 seconds) and evaluated using a single diode in the CCD-array. The signal-tonoise ratios of these six densitograms (using the atrazine-peak) where measured. The more the bit-range measurement increases, the more the linear signal-to-noise ratio increases as well. This is also plotted in figure 3.1-2 and shows that the signal-to-noise ration increases directly linear with the measuring time. Different amounts of atrazine were separated, stained and measured using 6 seconds measuring time and 64 densitograms bundled. The result is plotted in figure 3.1-3. The densitograms can be used to quantify atrazine in a strictly linear calibration range of two magnitudes. To obtain linearity, the camera must show a linear response curve. Moreover it is absolutely necessary to use the correct transformation algorithm for calculating absorption values.

\section{References}

[1] Vovk I., Prošek M., Kaiser R. E.: Image analysis, 464-488, in Sz. Nyiredy (editor) Planar chromatography. A retrospective view for the third millennium, Springer Scientific Publisher 2001, Budapest

[2] Aldridge P. K., Callis J. B., Burns D. H.: Laptop chemistry: A compact portable thin layer scanner, J. liquid Chromatogr. 1990, 13, 2829-2839

[3] Cosgrove J. A., Bilhorn R. B.: Spectrometric analysis of planar separations using charged-coupled device detection, J. Planar Chromatogr. 1989, 2, 362-367

[4] Brown S. M., Busch K. L.: A chargecoupled device for optical detection of sample bands in thin-layer-chromatograms, J. Planar Chromatogr. $1992,5,338-342$

[5] Pollak V. A., Doelemeyer A., Winkler W., Schulze-Clewing J.: Important design features of a system for the densitometric analysis of twodimensional flat-bed separations, J. Chromatogr. 1992, 596, 241-249

[6] Stroka J., Peschel T., Tittelbach G., Weidner G., van Otterdijk R., Anklam E.: Modification of an office scanner for the determination of aflatoxins after TLC separation, J. Planar Chromatogr. 2001, 14, 109 112
[7] Spangenberg B., Stehle S., Ströbele Ch.: Quantitative DC mit einem Handscanner: $\mathrm{CO}_{2}$-Bestimmung", GIT 1995, 39, 461 - 464

[8] Delley R., Friedrich K., Karlhuber G., Székely G., Stammbach K.: The identification and determination of various triazine herbicides in biological materials, Z. Anal. Chem. $1967,228,23-38$

[9] Rydon H. N., Smith P. W. G.: A new method for the detection of peptides and similar compounds on paper chromatography, Nature 1952, 169 , $922-923$

[10] Balinova A.: Thin-layer chromatographic detection of some systemic fungicides and their metabolites, J. Chromatogr. 1975, 111, 197 I199

[11] Székely G., Weick P., Abt B.: Determination of atrazine traces in ground and drinking water by thin layer chromatography, J. Planar Chromatogr. 1989, 2, 321 - 322

[12] Jork H., Roth B.: Vergleichende chromatographischeUntersuchung bei s-Triazinen, J. Chromatogr. 1977, 144, $39-56$

[13] Spangenberg B.: Does the KubelkaMunk theory describe TLC evaluations correctly? J. Planar Chromatogr., 2006, 19, $332-341$

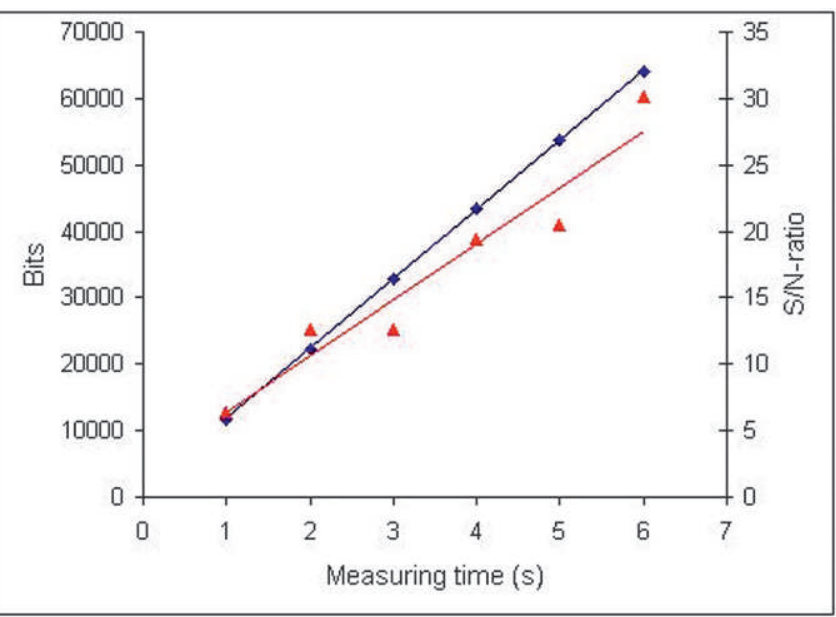

Fig. 3.1-2: Plotted is the response curve of the ST-1603 ME CCD-camera (bits against measuring time) and the obtained signal-to-noise ratios (S/N-ratios) plotted as triangles

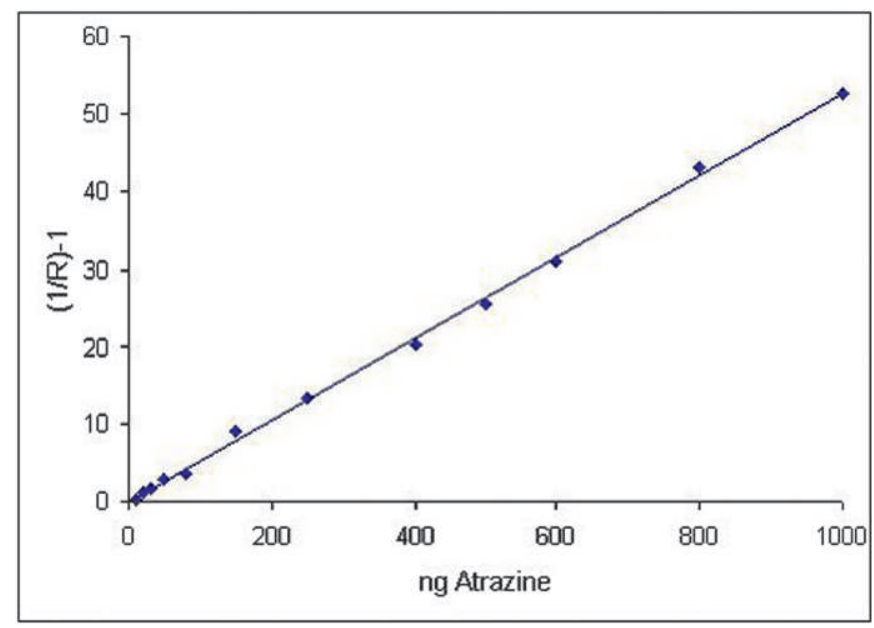

Fig. 3.1-3: Plotted is the range of linearity for atrazine from $10 \mathrm{ng}$ to $1000 \mathrm{ng}$ 